\title{
The CMS Tracker upgrade for HL-LHC
}

\section{Sudha Ahuja on behalf of the CMS Collaboration*t}

UNESP - Universidade Estadual Paulista (BR)

E-mail: sudha.ahuja@cern.ch

The LHC machine is planning an upgrade program which will smoothly bring the luminosity to about $5 \times 10^{34} \mathrm{~cm}^{-2} \mathrm{~s}^{-1}$ in 2028 , to possibly reach an integrated luminosity of $3000 \mathrm{fb}^{-1}$ by the end of 2037. This High Luminosity LHC scenario, HL-LHC, will require a preparation program of the LHC detectors known as Phase-2 upgrade. The current CMS Outer Tracker, already running beyond design specifications, and CMS Phase-1 Pixel Detector will not be able to survive HLLHC radiation conditions and CMS will need completely new devices, in order to fully exploit the high-demanding operating conditions and the delivered luminosity. The new Outer Tracker should have also trigger capabilities. To achieve such goals, R\&D activities are ongoing to explore options both for the Outer Tracker, and for the pixel Inner Tracker. Solutions are being developed that would allow including tracking information at Level-1. The design choices for the Tracker upgrades are discussed along with some highlights of the R\&D activities.

The European Physical Society Conference on High Energy Physics

5-12 July, 2017

Venice

\footnotetext{
* Speaker.

${ }^{\dagger}$ This material is based upon work supported by the Sao Paulo Research Foundation (FAPESP) under Grant No.
} 2013/01907-0. 


\section{Introduction}

The Large Hadron Collider (LHC) has shown excellent performance since the start of operation in 2009. LHC experiments have been able to deliver highly relevant physics results using the high quality proton-proton (pp) collision data. The rate increased steadily achieving instantaneous luminosities of up to $1.5 \times 10^{34} \mathrm{~cm}^{-2} \mathrm{~s}^{-1}$ in 2016 , exceeding the LHC design value. The main preparation of the High Luminosity phase of the LHC (HL-LHC) will start around 2024 [1] (Long Shutdown 3 (LS3)). The accelerator will be upgraded to handle peak luminosities of up to 5/7.5 $\times 10^{34} \mathrm{~cm}^{-2} \mathrm{~s}^{-1}$, allowing the experiments (ATLAS/CMS) to collect integrated luminosities of up to $3000 \mathrm{fb}^{-1}$. This will also be accompanied by an upgrade of the experiments to maintain the excellent performance of the detector and fully utilize the capabilities of the HL-LHC, along with dealing with the challenging radiation environment and operating conditions. The CMS detector will require improved radiation hardness, higher detector granularity to reduce occupancy, increased bandwidth to accommodate higher data rates, and an improved trigger capability in order to maintain an acceptable trigger rate while not compromising physics potential [2]. The entire tracking system will need to be replaced to deal with the HL-LHC environment.

\section{Tracker for HL-LHC}

The current strip tracker was designed to operate efficiently at an instantaneous luminosity of up to $1 \times 10^{34} \mathrm{~cm}^{-2} \mathrm{~s}^{-1}$, with performance degradation beyond $500 \mathrm{fb}^{-1}$. The original pixel detector has already been replaced by a new Phase-1 pixel detector [3] during the extended year-end technical stop (EYETS) 2016/2017. Both the strip tracker and the Phase-1 pixel detector will have to be replaced before HL-LHC operation, to deal with the extreme operational conditions. Along with increased radiation tolerance and detector granularity, the Phase- 2 tracker will also require an improved two-track separation, reduced material budget, robust pattern recognition, level-1 track trigger capability, and an extended tracking acceptance (up to $\eta<4.0$ ). Figure 1 shows the sketch of one quarter of the tracker layout in the $r-z$ view. In the Inner Tracker, the yellow and green lines correspond to pixel modules made of two and four readout chips, respectively. The blue and red lines correspond to the two types of modules of the Outer Tracker. The following sub-sections will describe the Inner and Outer Tracker in more detail.

\subsection{Inner Tracker}

The Phase-2 Inner Tracker (IT) [1] is designed to maintain or improve the tracking and vertexing capabilities under the high pileup (140 - 200 collisions per bunch crossing) conditions of the HL-LHC. The IT will be built from pixel modules with thin silicon sensors (thickness 100 - 150 $\mu \mathrm{m}$ ), segmented into pixel sizes of $25 \times 100 \mu \mathrm{m}^{2}$ or $50 \times 50 \mu \mathrm{m}^{2}$. They have to deal with high radiation dose (up to $1.2 \mathrm{Grad}$ ) and hit rates (approaching $3 \mathrm{GHz} / \mathrm{cm}^{2}$ in the inner layers). They are expected to exhibit the required radiation tolerance and to deliver the desired performance in terms of detector resolution, occupancy, and two-track separation. IT is composed of a barrel part with four cylindrical layers and eight small plus four large disc-like structures in each forward direction. The IT is extractable, i.e., there is a possibility to extract and replace the degraded parts of the detector without removing the beam pipe. The Tracker Endcap Pixel detector (TEPX), installed 


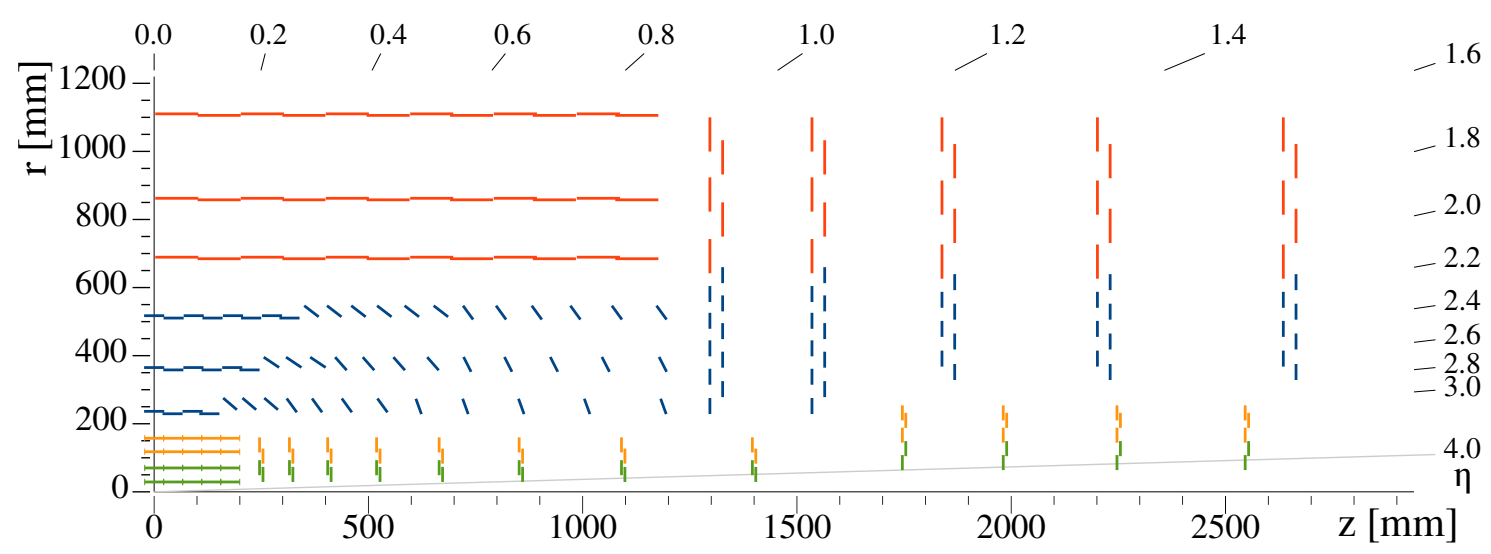

Figure 1: Phase-2 tracker layout in $r-z$ view [1].

within the extended space, will enable the measurement of real-time instantaneous luminosity as an added functionality. The extended geometrical coverage of up to $\eta<4.0$ provides large forward acceptance to mitigate pileup (in particular in the endcap calorimeters). Figure 2 shows the pictorial representation of the IT sensor modules with $1 \times 2$ and $2 \times 2$ pixel readout chips (PROC) and also the occupancy map as a function of $\eta$ for each layer from the barrel and the endcap discs. A common development is being carried out for the PROC by both CMS and ATLAS in the RD53 collaboration [4].
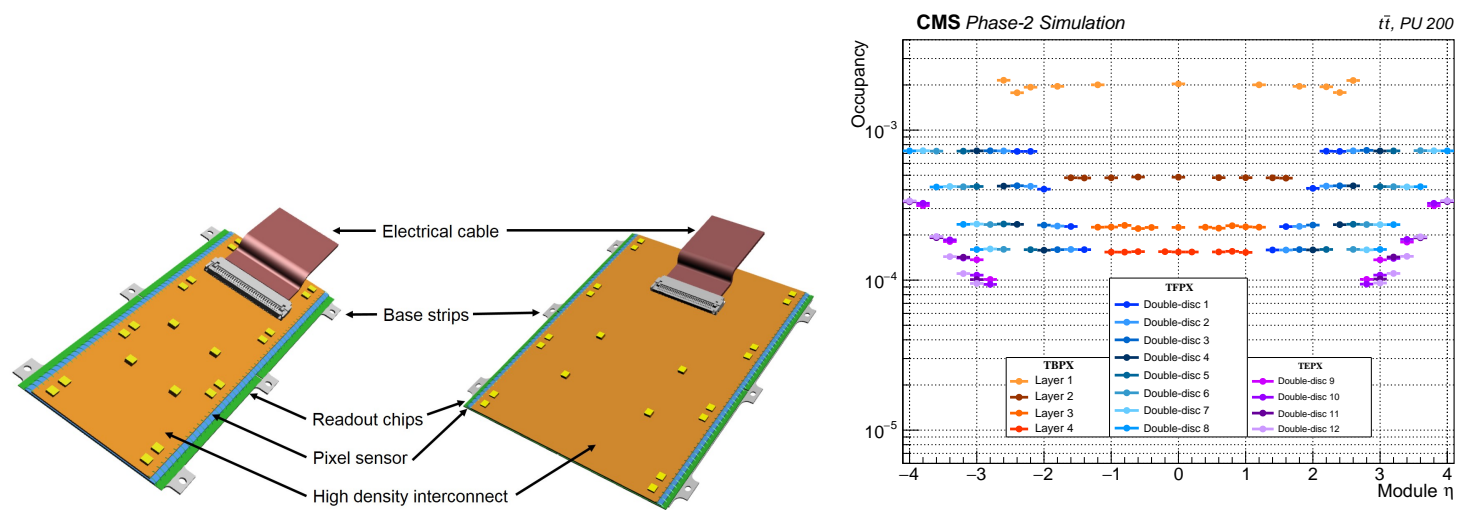

Figure 2: IT modules and occupancy map [1].

\subsection{Outer Tracker}

The Outer Tracker (OT) [1] is composed of six cylindrical barrel layers in the central region, covering the region of $|z|<1200 \mathrm{~mm}$, with five endcap double-discs on each side, in the region of $1200<|z|<2700 \mathrm{~mm}$. The OT is populated with $p_{T}$ modules, implementing the Level-1 (L1) trigger functionality. Two versions of $p_{T}$ modules will be used in the OT, modules with two strip sensors (2S) and modules with a strip and a macro-pixel sensor (PS). The PS modules provide precise $z$-coordinate measurement and are placed in the first three layers of the OT, in the radial region of 200-600 $\mathrm{mm}$. The $2 \mathrm{~S}$ modules are deployed in the outermost three layers, in the radial 
region above $600 \mathrm{~mm}$. The modules in the endcaps are arranged in rings on disc-like structures, with rings at low radii (up to $700 \mathrm{~mm}$ ) using PS modules and $2 \mathrm{~S}$ modules being used at larger radii. Figure 3 shows the OT sensor modules along with the occupancy map as a function of $\eta$. Data from the modules is read out by front end chips: CMS Binary Chip (CBC) in the 2S modules, Short Strip ASIC (SSA), and Macro-Pixel ASIC (MPA) in PS modules. Tracking at L1 is challenging due to the high data rates and tight latency requirements imposed. The sensors are read out by a common front end which correlates their signals and form hit pairs, also referred to as stubs, only above a certain $p_{T}$ threshold $(>2 \mathrm{GeV} / \mathrm{c}$ (programmable)). Data rates can be reduced by a factor of 10-100 by the on-detector $p_{T}$ filtering capability. These stubs are further used to reconstruct L1 tracks. The inner three layers in the barrel are arranged in a partially tilted geometry (Figure 1) to help mitigate stub inefficiency. The tracking trigger will have approximately $4 \mu$ s to reconstruct and deliver L1 tracks to a downstream processing system, which will use these tracks together with trigger primitives from the calorimeter and muon sub-detectors to perform physics objects reconstruction. The three L1 track finding approaches under study are Associative Memory (AM), Tracklet, and Time Multiplexed Track Trigger (TMTT). The AM approach uses a combination of Associative Memory ASICs and FPGAs to perform real time pattern recognition, while the other two rely solely on FPGAs. A solution based purely on FPGAs is presently being considered as the baseline for the L1 tracking system.
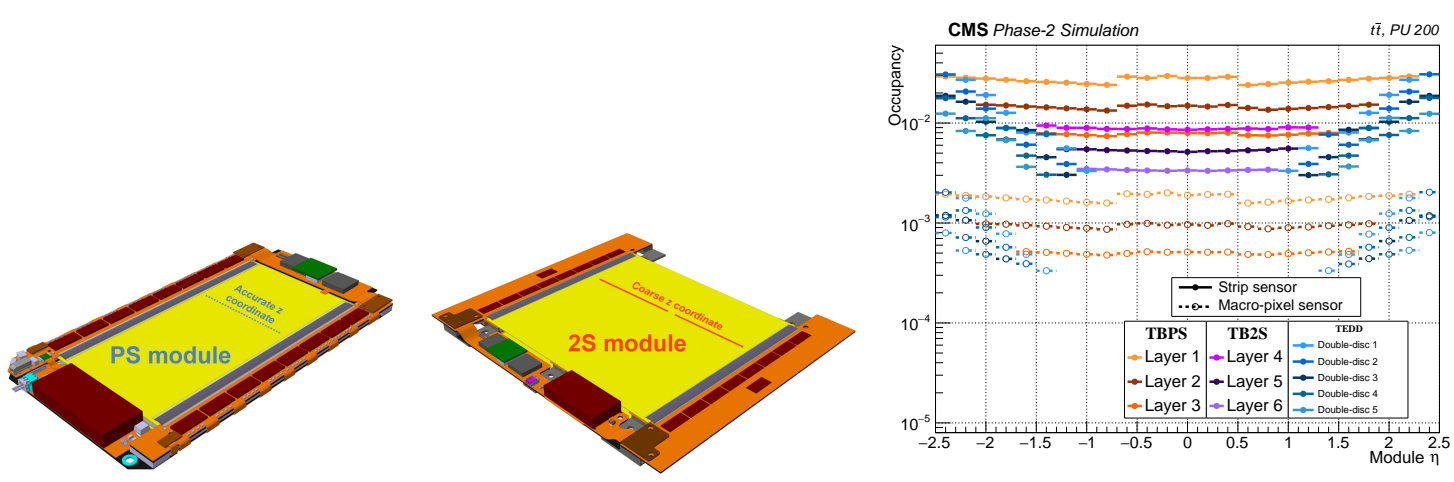

Figure 3: OT modules and occupancy map [1].

Figure 4 shows the stub reconstruction efficiency (left) for simulated muons with $1<p_{T}<10$ $\mathrm{GeV}$ and the L1 tracking efficiency (right) for prompt muons and electrons for $t \bar{t}$ events (pileup 200) with a sharp turn-on at $3 \mathrm{GeV}$ for muons and a slow turn-on for electrons.

\section{Offline Tracking Performance}

The CMS tracker has to maintain excellent offline tracking performance even during the challenging HL-LHC phase, where around 6000 charged particles will traverse the tracker each bunch crossing, produced by 200 collisions on average. Figure 5 (left) shows the offline tracking efficiency for single muons. The efficiency is stable in the entire range of pseudorapidity, in both pileup scenarios. The transverse momentum resolution for single muons with $p_{T}=10 \mathrm{GeV}$ can be seen in Figure 5 (right), for both the current and the Phase- 2 tracker. The improvement in Phase-2 

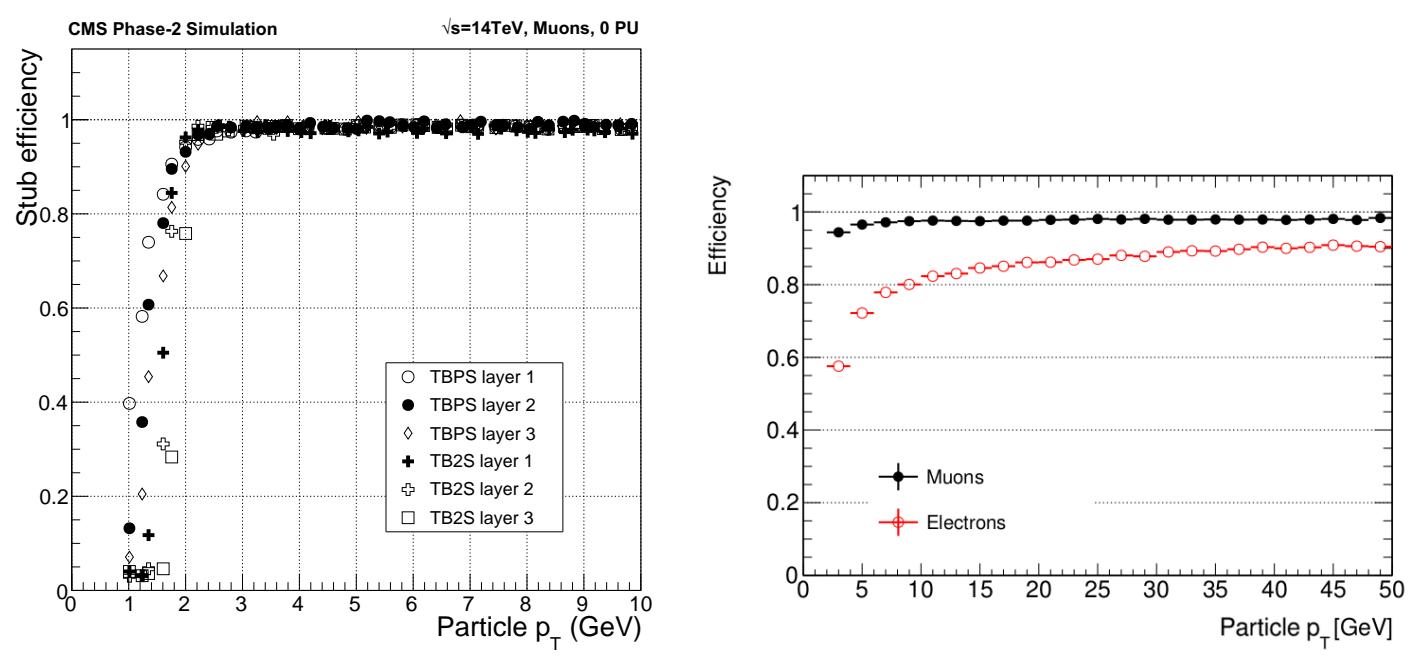

Figure 4: Stub reconstruction and L1 tracking efficiency [1].

can be attributed to better hit resolution and the reduction of the material budget of the upgraded detector.
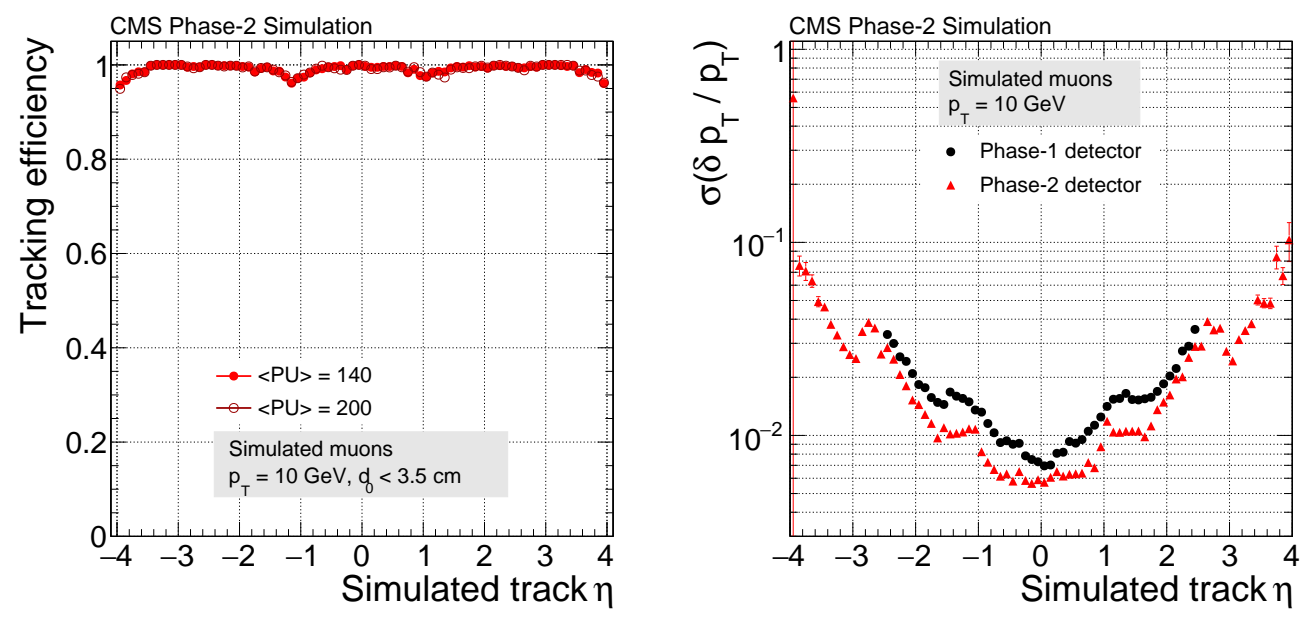

Figure 5: Offline tracking performance [1].

\section{Summary}

The excellent tracking performance represents one of the major tools to achieve a global event description that can stand the very high pileup scenarios. It will lead to an excellent performance of the pileup mitigation algorithms, b-tagging capability at high $\eta$, efficient jet substructure techniques up to $2 \mathrm{TeV}$, and even good tau identification consistent with pileup. It also sets the stage for the exploration of the multi- $\mathrm{TeV}$ scale physics using the large expected integrated luminosity collected during the nominal HL-LHC running scenarios. The large dataset also allows us to improve our understanding of the particle landscape. Thus, the upgraded tracker will pay a crucial role in 
enhancing the capabilities of the detector, which will extend its sensitivity to probe physics while maintaining the excellent performance achieved in Run 2. The Phase-2 Tracker upgrade program has a lot more ground to cover in the coming years.

\section{References}

[1] CMS Collaboration, The Phase-2 Upgrade of the CMS Tracker, CERN-LHCC-2017-009, CMS-TDR-014.

[2] CMS Collaboration, Technical Proposal for the Phase-II Upgrade of the CMS Detector, CERN-LHCC-2015-010, LHCC-P-008, CMS-TDR-15-02 (2015).

[3] CMS Collaboration, CMS Technical Design Report for the Pixel Detector Upgrade, CMS Technical Design Report CERN-LHCC-2012-016, CMS-TDR-11, 2012.

[4] RD53 Collaboration, RD Collaboration Proposal: Development of pixel readout integrated circuits for extreme rate and radiation, Scientific Committee Paper CERN-LHCC-2013-008, LHCC-P-006, 2013.

[5] CMS Collaboration, CMS Phase II Upgrade Scope Document, Technical Report CERN-LHCC-2015-019, LHCC-G-165, CERN, Geneva, 2015. 